\title{
Downregulation of miR203 induces overexpression of PIK3CA and predicts poor prognosis of gastric cancer patients
}

This article was published in the following Dove Press journal:

Drug Design, Development and Therapy

I6 July 2015

Number of times this article has been viewed

\author{
Min Liangl,* \\ Boyun Shi ${ }^{1, *}$ \\ Jifang Liu' \\ Lu He ${ }^{2}$ \\ Gao Yi' \\ Lin Zhou' \\ Guifang Yu' \\ Xinke Zhou',* \\ 'Department of Oncology, \\ The fifth affiliated hospital of \\ Guangzhou Medical University, \\ Guangzhou, Guangdong, People's \\ Republic of China; ${ }^{2}$ Department \\ of radiotherapy, Affiliated Cancer \\ Hospital of Guangzhou Medical \\ University, Guangzhou, Guangdong, \\ People's Republic of China \\ *These authors contributed equally to \\ this work
}

Background: Despite advances in clinical therapies and technologies, the prognosis for patients with gastric cancer is still poor. The aim of this study is to investigate new predictive markers for prognosis of gastric cancer.

Methods: In this study, we evaluated the expression pattern of PIK3CA in 107 gastric cancer specimens and their adjacent nontumorous tissues. PIK3CA siRNA was synthesized and transfected into gastric cancer cell lines. Colony formation and MTT assays were employed to analyze the cell proliferation. PIK3CA expression was examined by using immunohistochemical analysis and Western blot assay. Transwell invasion assay was used to detect the invasion capability of the cells. Luciferase activity was examined by using 3 '-untranslated region luciferase reporter assays.

Results: We observed that $P I K 3 C A$ was significantly upregulated in gastric cancer tissues. High expression level of PIK3CA was detectable in 48 (44.86\%) of the gastric cancer specimens, and correlated with poor prognosis. In addition, our study indicated that miR203 inhibits cell proliferation and invasion via directly targeting and suppressing the PIK3CA expression. MiR203 expression is downregulated in gastric cancer tissues. Moreover, low expression level of miR203 predicted poor prognosis of gastric patients and induced overexpression of $P I K 3 C A$. Our further study also reported that overexpression of miR203 inhibited phosphorylation of AKT, while cotransfection of PIK3CA reversed the effect of miR203.

Conclusion: Our study suggested a miR203-PIK3CA-AKT signaling pathway in gastric cancer cells. This signaling pathway might play an important role in gastric cancer genesis and development.

Keywords: AKT, gastric cancer, miR203, PIK3CA, prognosis

\section{Introduction}

Gastric cancer is the second leading cause of cancer-related deaths worldwide. The incidence of gastric cancer is comparatively higher in the Eastern Asia, and 65\%-70\% of new cases of deaths are reported from gastric cancer in less-developed countries. ${ }^{1}$ In 2009, the incidence rate of gastric cancer ranked third among the most common cancers in People's Republic of China. ${ }^{2}$ Currently, surgery is one of the most common treatments of gastric cancer but survival rate of patients is less than 33\%.,4 Thus, there is an immense need to identify novel and promising therapeutic target for the cure and treatment of gastric cancer. Previous studies have revealed several genes related to human gastric cancer, but successful therapeutic targets are limited. Thus, the molecular mechanisms of gastric cancer are of great importance and remain to be elucidated.

PIK3CA gene is located at 3q26.3 and encodes the key enzymatic subunit p1 $10 \alpha$ of phosphatidylinositol 3-kinase (PI3K). ${ }^{5}$ PI3K signaling pathway plays an 
important role in carcinogenesis. ${ }^{6} \mathrm{PIK} 3 \mathrm{CA}$ functions as an oncogene, which plays important roles in many cancers, including gastric cancer ${ }^{7-9}$ and colorectal cancer. ${ }^{10-12}$ Our previous studies have demonstrated that overexpression of PIK3CA increased invasion and proliferation of gastric cancer cells. ${ }^{13}$ Moreover, the increased expression level of $P I K 3 C A$ in gastric cancer tissues is associated with lymph node metastasis. ${ }^{13,14}$

On this basis, we investigate the upstream and downstream molecules of PIK3CA and identify that PIK3CA is a novel target of miR203 in gastric cancer. A decrease in miR203 expression upregulates $P I K 3 C A$, and thus activates $\mathrm{PI} 3 \mathrm{~K} / \mathrm{AKT}$ signaling pathway and increases invasion and proliferation of gastric cancer cells. Moreover, we also demonstrate that low expression level of miR203 correlates with high expression level of $P I K 3 C A$, and both decreased miR203 expression and increased $P I K 3 C A$ expression correlate with poor prognosis of gastric cancer patients.

\section{Materials and methods}

\section{Patients and specimens}

The study was approved by the Institutional Review Board and Human Ethics Committee of Affiliated Oncologic Hospital of Guangzhou Medical University. Written consent for using the samples for research purposes was obtained from all patients prior to surgery. We collected tissue from 107 patients with advanced gastric carcinoma who were treated at the Affiliated Oncologic Hospital of Guangzhou Medical University (Guangzhou, People's Republic of China) between June 2005 and December 2008. All the patients were monitored after surgery until September 31, 2012. A total of 107 gastric carcinoma samples (tumor and matched adjacent nontumorous tissues) were used in the immunohistochemistry (IHC) analysis.

\section{Cell lines, transfection, and antibodies}

The gastric cancer cell lines SGC-7901, BGC-823, MGC-803, and HGC-27 were cultured in RPMI 1640 (Thermo Scientific, Waltham, MA, USA) medium supplemented with $10 \%$ fetal bovine serum, $50 \mathrm{mg} / \mathrm{mL}$ streptomycin, and $50 \mathrm{U} / \mathrm{mL}$ penicillin in a humidified atmosphere containing $5 \% \mathrm{CO}_{2}$ at $37^{\circ} \mathrm{C}$. All antibodies used in this study were obtained from Santa Cruz Biotechnology (Santa Cruz, CA, USA). MiR203 mimics and miR203 antisense oligonucleotides (ASO) were purchased from Shanghai GenePharma (Shanghai, People's Republic of China). Transfections were carried out using Lipofectamine-2000 (Invitrogen, Carlsbad, CA, USA) according to the manufacturer's instructions.

\section{Immunohistochemical analysis}

Formalin-fixed, paraffin-embedded tissue specimens from gastric carcinoma patients were cut in $5 \mu \mathrm{m}$ sections. Briefly, after deparaffinized in xylene and rehydrated, the tissue slides were then treated with $3 \%$ hydrogen peroxide, and the antigens were retrieved in sodium citrate buffer ( $\mathrm{pH}$ 6.0) using a microwave oven. After 1 hour of preincubation in goat serum to prevent nonspecific staining, the specimens were incubated with primary antibodies overnight at $4{ }^{\circ} \mathrm{C}$. The tissue slides were treated with a non-biotin horseradish peroxidase detection system according to the manufacturer's instructions (DAKO, Glostrup, Denmark). Two different pathologists evaluated the results of IHC. Both the extent and intensity of immunostaining were taken into consideration. The intensity of staining was scored from 0 to 3 , and the extent of staining was scored from $0 \%$ to $100 \%$. The final score of each staining was obtained by multiplying the two scores. The pAKT expression was recognized as higher level, if the score was higher than 1.5; if the score was 1.5 or less, the case was classified as low expression. PIK3CA expression was considered high if the score reached 0.5 .

\section{Colony formation and MTT assays}

SGC-7901 and HGC-27 cells in the logarithmic growth phase were planted into six-well plate with 200 cells per well. The cells were continuously cultured for 9 days. The RPMI-1640 medium was then discarded. The cells were washed with phosphate buffered saline (PBS; pH 6-8) twice, fixed with methyl alcohol for 20 minutes, stained with 10\% Giemsa stain for 20 minutes, washed with distilled water, and then dried. Colonies containing more than 50 cells were counted. For MTT assay, 1,000 cells were seeded into a 96-well plate and cultured for 24, 48, and 72 hours. The MTT was then added (concentration of $5 \mathrm{mg} / \mathrm{mL}, 10 \mu \mathrm{L} /$ well). The cells were then cultured for 4 hours and centrifuged at 1,000 rpm for 5 minutes. The supernatant liquid was discarded. About $150 \mu \mathrm{L}$ of DMSO was added per well, followed by agitating for 10 minutes to completely dissolve the crystals. A wavelength of $570 \mathrm{~nm}$ was selected, and the optical density per well was detected.

\section{Transwell invasion assay}

Transwell invasion assay was performed using the BD BioCoat Matrigel Invasion Chamber (BD Bioscience, San Jose, CA, USA). Briefly, $2 \times 10^{4}$ cells were placed into the top chamber of each insert and incubated at $37^{\circ} \mathrm{C}$ for 48 hours. Cells that invaded through the Matrigel were stained using crystal violet (Sigma-Aldrich Co., St Louis, MO, USA) and quantified. 


\section{RNA extraction and real-time PCR}

Reverse transcription polymerase chain reaction (RT-PCR) was performed using TaqMan ${ }^{\circledR}$ MicroRNA Reverse Transcription Kit and TaqMan ${ }^{\circledR}$ Universal PCR Master Mix (Ambion, Austin, TX, USA). For analysis of miR203 expression (U6 small nuclear RNA, a reference gene), we employed a two-step quantitative reverse transcription PCR (qRT-PCR) with specific primers for miR203 (we used the subclassification of miR-203-3p) (Applied Biosystems, Foster City, CA, USA) following the manufacturer's protocol. Real-time RT-PCR was performed using a PRISM 7300 Sequence Detection System (Applied Biosystems), in which each reaction $(25 \mu \mathrm{L})$ contained $10 \mu \mathrm{L}$ PCR Master Mix (Ambion) and $1.33 \mu \mathrm{L}$ RT product, and each sample was analyzed in triplicates. PCR was carried out at $95^{\circ} \mathrm{C}$ for 10 minutes, followed by 40 cycles of amplification at $95^{\circ} \mathrm{C}$ for 15 seconds and $60^{\circ} \mathrm{C}$ for 60 seconds. Results are representative of two independent assays. Relative fold changes of expression in tumor tissues were calculated using the comparative $\mathrm{Ct}$ $\left(2^{-\Delta \Delta \mathrm{Ct}}\right)$ method with U6 small nuclear RNA (Ambion) as the endogenous control.

\section{SiRNA synthesis and transfection}

The siRNAs were designed and synthesized by GenePharma. PIK3CA siRNA: sense $5^{\prime}$-GGC UAA AGA AAG CCU UUA UTT-3', antisense 5'-AUA AAG GCU UUC UUU AGC CTT-3'; negative control siRNA: sense 5'-UUC UCC GAA CGU GUC ACG UTT-3', antisense 5'-ACG UGA CAC GUU CGG AGA ATT- $3^{\prime}$. All sequences were submitted to National Institutes of Health Blast program to ensure gene specificity.

\section{Vector construct}

The pcDNA-PIK3CA was used as a template. PCR amplification was performed by using a standard protocol with the primers: sense $5^{\prime}$-GGC UAA AGA AAG CCU UUA UTT- $3^{\prime}$, antisense $5^{\prime}$-AUA AAG GCU UUC UUU AGC CTT-3'; Negative control siRNA: sense 5'-UUC UCC GAA CGU GUC ACG UTT-3', antisense 5'-ACG UGA CAC GUU CGG AGA ATT- $3^{\prime}$. The gene was purified and inserted into pcDNA3.1 vector. The recombinant plasmid (pcDNA-PIK3CA) was confirmed by sequencing.

\section{3'-UTR luciferase reporter assays}

Firefly luciferase reporter plasmids, pMIR-REPORT (Ambion) containing $3^{\prime}$-untranslated region (UTR) and 3'-UTR-mut of the PIK3CA gene or empty luciferase vector obtained from Ambion were used for luciferase reporter assay. Cells were seeded in a 48-well plate and were cotransfected with miR203 mimics, ASO as well as PIK3CA-3'UTR or PIK3CA-3'UTR-mut. Luciferase activity was analyzed 24 hours post-transfection using the Luciferase Reporter Assay System (Promega, Madison, WI, USA) following the manufacturer's instructions.

\section{Western blot}

The specimens were snap-frozen in liquid nitrogen and immediately homogenized in ice-cold RIPA lysis buffer (Sigma-Aldrich Co.). Denatured total tissue lysates were run in sodium dodecyl sulfate polyacrylamide gel electrophoresis (SDS-PAGE), transferred to nitrocellulose membranes, and blocked overnight at $4^{\circ} \mathrm{C}$ in $5 \%$ milk. The blocked membranes were incubated overnight at $4{ }^{\circ} \mathrm{C}$ with the PIK3CA monoclonal antibody (Santa Cruz, 1:4,000), pAKT monoclonal antibody (Santa Cruz, 1:3,000), the AKT monoclonal antibody (Santa Cruz, 1:3000), and $\beta$-actin monoclonal antibody (Santa Cruz, 1:2,000) followed by 1 hour incubation at room temperature with an HRP (horseradish peroxidase)-conjugated secondary antibody (Santa Cruz, 1: 1,000). Immunodetection was performed using the ECL+ reagents (Amersham Biosciences, Buckinghamshire, UK). Signals were normalized against $\beta$-actin and the results were expressed as a percentage of the negative control signal.

\section{Statistics}

All statistical analyses were performed with SPSS statistical software (version 21.0; SPSS, IBM, Armonk, NY, USA). Survival curves were constructed using the Kaplan-Meier method and analyzed by the log-rank test. The two-tailed chi-squared test was used to compare count data. The Student's $t$-test was used for comparisons. $P$-values $<0.05$ were considered to be statistically significant.

\section{Results}

\section{Overexpression of PIK3CA indicates poor prognosis of gastric patients}

In our previous study, ${ }^{8}$ we have demonstrated that $P I K 3 C A$ could increase proliferation and invasion of gastric cancer cells. Hence, we detected expression level of PIK3CA in gastric cancer and adjacent nontumorous tissues. By using IHC assays, we detected high expression of PIK $3 C A$ in 48/107 gastric cancer tissues, compared with only 22/107 in adjacent nontumorous tissues $(P<0.001$; Figure $1 \mathrm{~A}$ and $\mathrm{B})$. Further statistical analysis indicated that high expression of $P I K 3 C A$ predicted poor prognosis for disease-free survival (DFS) $(P=0.003)$ and overall survival $(\mathrm{OS})(P=0.001)$ in 

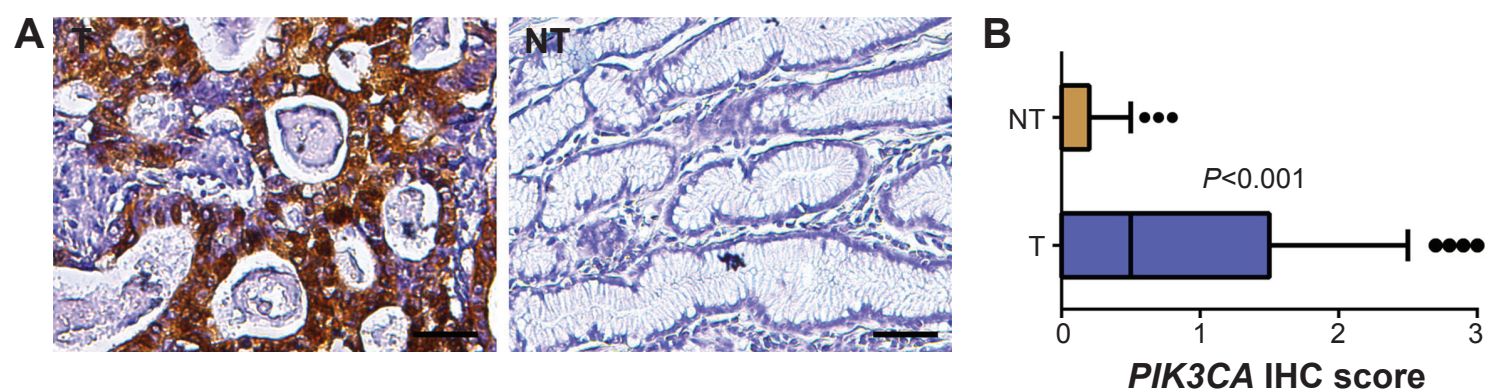

C

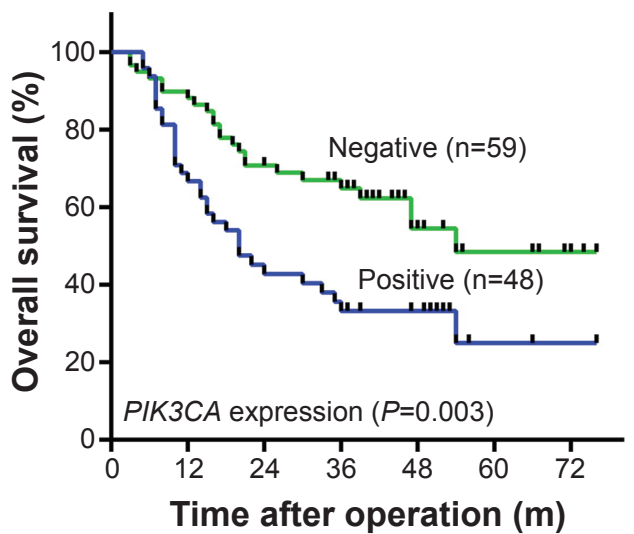

D

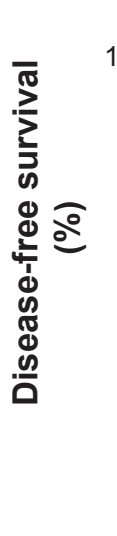

PIK3CA IHC score

Figure I PIK3CA was significantly upregulated in gastric carcinoma and was prognostic for OS and DFS in gastric carcinoma.

Notes: (A) Immunohistochemistry (IHC) assays of PIK3CA expression in 107 paired gastric carcinoma and adjacent nontumorous tissues. Scale bars, $50 \mu \mathrm{m}$. (B) Box plot graph showing PIK3CA expression level compared among gastric carcinoma and adjacent nontumorous tissues. (C, D) Prognostic significance assessed by Kaplan-Meier survival estimates and log-rank tests. Comparison of OS and DFS by PIK3CA. $P<0.05$.

Abbreviations: OS, overall survival; DFS, disease-free survival; T, tumorous tissues; NT, nontumorous tissues.

gastric cancer. Disease-free survival and overall survival of the group showing high expression of PIK3CA were significantly lower than the group showing low expression (Figure 1C and D).

\section{PIK3CA is a novel target of miR203}

Overexpression of PIK3CA indicated poor prognosis of gastric cancer patients; however, the mechanism of aberrant $P I K 3 C A$ expression in gastric cancer is still unclear. By performing bioinformatics analysis, we supposed that PIK3CA is a potential target of miR203 (Figure 2A, up panel). To verify this correlation, we performed luciferase assays and found that miR203 could inhibit luciferase activity of PIK3CA 3'UTR-Luc vector transfected group but not PIK3CA 3'UTRmutated-Luc vector group (Figure 2A, down panel). Then we tested expression levels of miR203 and PIK3CA in four gastric cancer cells. The results illustrated that there was a negative trend of PIK3CA expression in cells showing high expression of miR203 (Figure 2B and C). Moreover, the transfection of miR203 mimics and miR203 ASO indicated that miR203 mimics could decrease PIK3CA expression in PIK3CA high-expression gastric cell line, HGC-27. However, miR203 ASO could increase PIK3CA expression in
SGC-7901, a miR203 high-expression but PIK3CA lowexpression cell line (Figure 2D).

Next, we analyzed the influence of miR203 on proliferation, colony formation, and invasion of gastric cancer cells. We found miR203 mimics could decrease proliferation, colony formation, and invasion of gastric cancer cells; however, miR203 ASO could increase proliferation, colony formation, and invasion of gastric cancer cells (Figure 2F-I). To further confirm the hypothesis that miR203 exerts anti-cancer effect due to inhibition of PIK3CA expression, we designed siRNA targeted to $P I K 3 C A$ and constructed $P I K 3 C A$ expression vector (Figure 2E). The cotransfection indicated that PIK3CA siRNA and expression vector reversed the effect of miR203 mimics and miR203 ASO (Figure 2F-I). These experiments demonstrated that miR203 targeted and inhibited PIK3CA expression. Moreover, miR203 exerted its anti-cancer effect via inhibition of PIK3CA expression.

\section{PIK3CA expression is associated with miR203 and phosphorylation of AKT}

To further confirm expression profile of miR203 in gastric cancer tissues, we performed real-time quantitative PCR in 107 cases of gastric cancer and adjacent nontumorous tissues. 
In general, an average three- to fourfold decrease for miR203 expression was noted in gastric cancer tissues as compared with that of adjacent nontumorous tissues (Figure 3A). Comparative analysis of paired gastric cancer tissues with adjacent nontumorous tissues further revealed that reduced
miR203 expression (more than twofold) was observed in $85(79.42 \%)$ cases, suggesting that reduction of miR2 203 was a frequent event in human gastric cancer. Furthermore, we found that miR203 expression level was negatively correlated with PIK3CA expression (Figure 3B). This result verified

A gcacuuaacuaguucauuuca pik3CA-3Utr

GAUCACCAGGAUUUGUAAAGUG hsa-miR203

GCACUUAAACUAGUUGAUUUGA PIK3CA-3UTR-mut

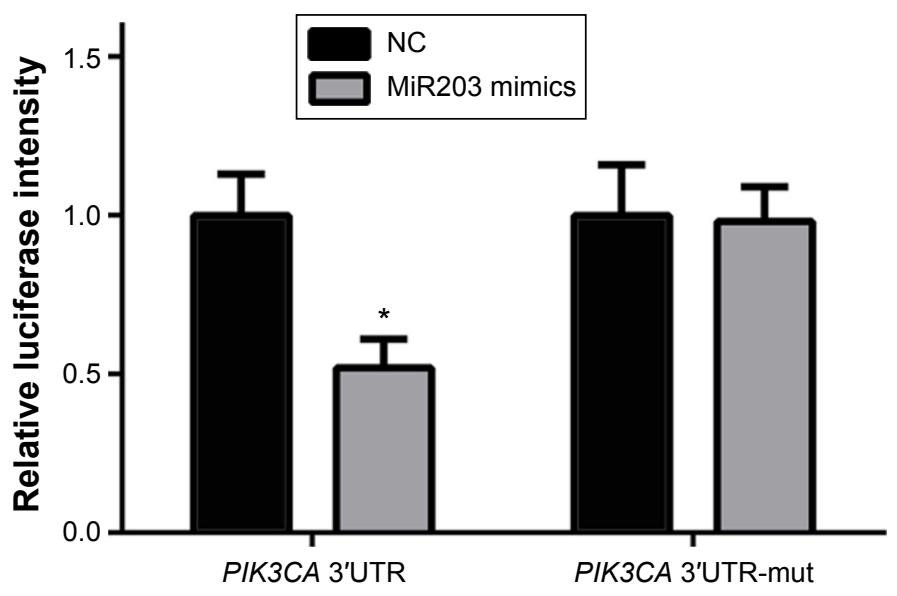

B Relative expression level of miR203

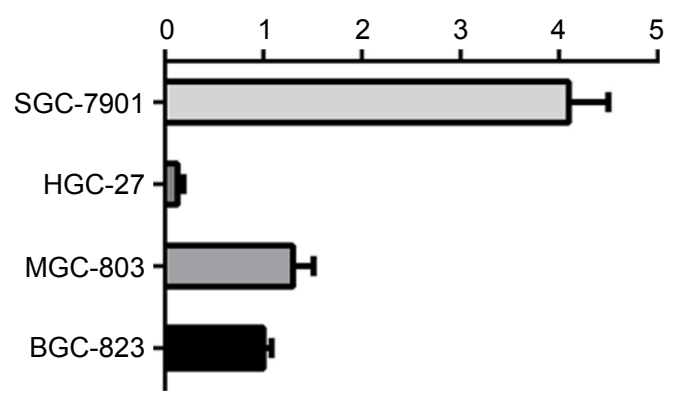

D

HGC-27 SGC-7901

C

E

HGC-27

SGC-7901

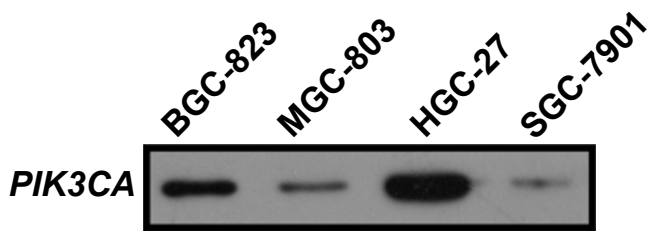

PIK3CA

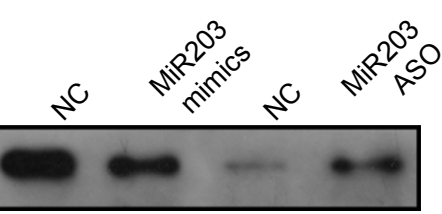

$\beta$-actin

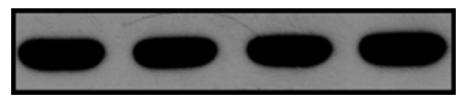

$\beta$-actin

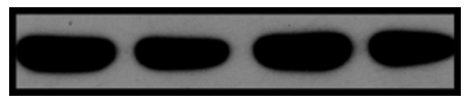

$\beta$-actin

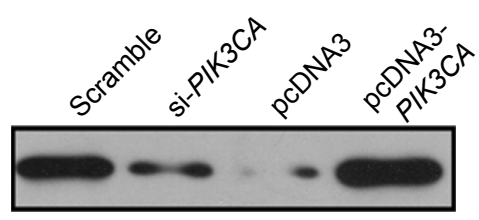

Figure 2 (Continued) 

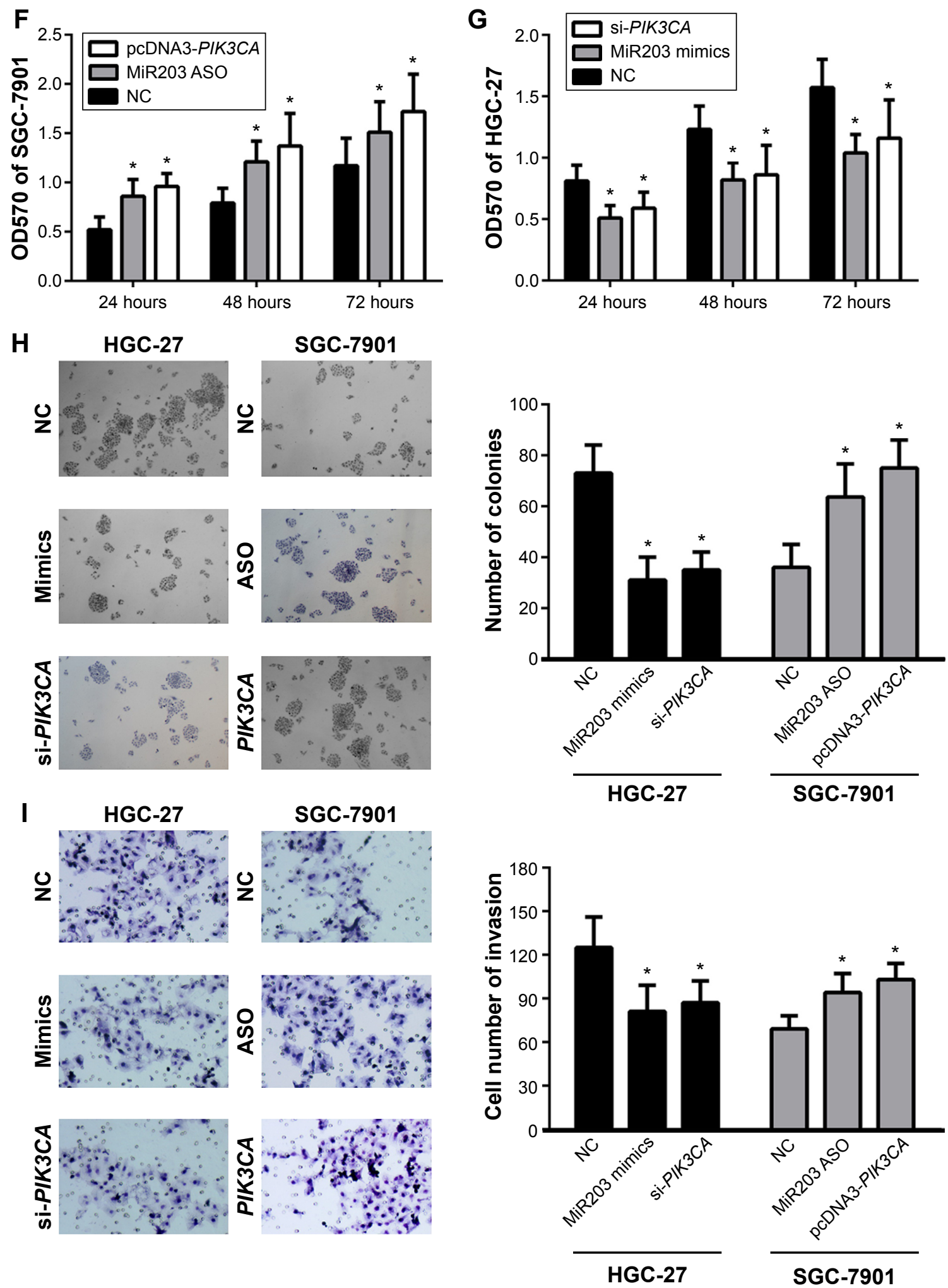

Figure 2 MiR203 expression level correlated with the expression of PIK3CA, and significantly decreased gastric cancer cell proliferation and invasion. Notes: (A) Luciferase reporter gene assay indicated that miR203 targeted PIK3CA. (B) The miR203 expression pattern in gastric cancer cells. (C) The PIK3CA expression pattern in gastric cancer cells. (D) Overexpression of miR203 in HGC-27 decreased PIK3CA expression and knockdown of miR203 in SGC-790I increased PIK3CA expression, this result indicated that miR203 expression negatively correlated with PIK3CA. (E) Overexpression of PIK3CA in SGC-790I and knockdown of PIK3CA in HGC-27. (F) MTT assay suggested that miR203 mimics inhibited proliferation of gastric cancer cells partly due to downregulating PIK3CA expression. (G) MTT assay suggested that miR203 ASO increase proliferation of gastric cancer cells via upregulating PIK3CA. (H) Clone formation assays suggested that miR203 inhibited clone formation capacity by downregulating PIK3CA. (I) Transwell assays indicated that miR203 decreased invasion of gastric cancer cells by targeting PIK3CA. $* P<0.05$ represents the values compared to the NC group. Abbreviations: UTR, untranslated region; NC, normal control; ASO, antisense oligonucleotides; OD, optical density. 

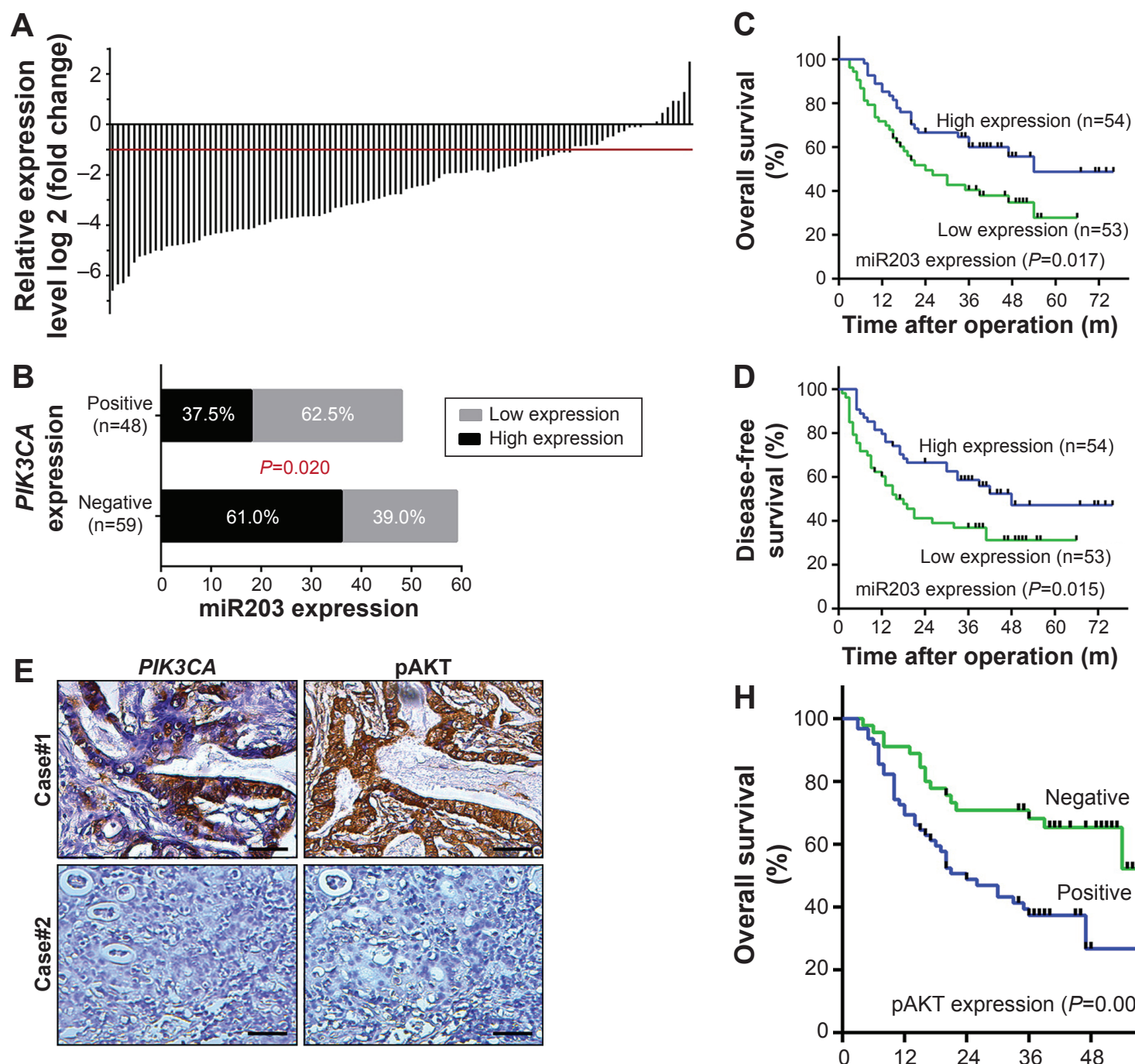

Time after operation $(\mathrm{m})$

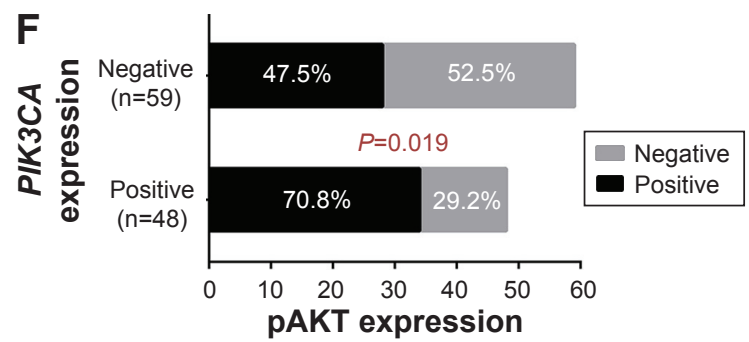

G $\quad$ HGC-27 SGC-7901
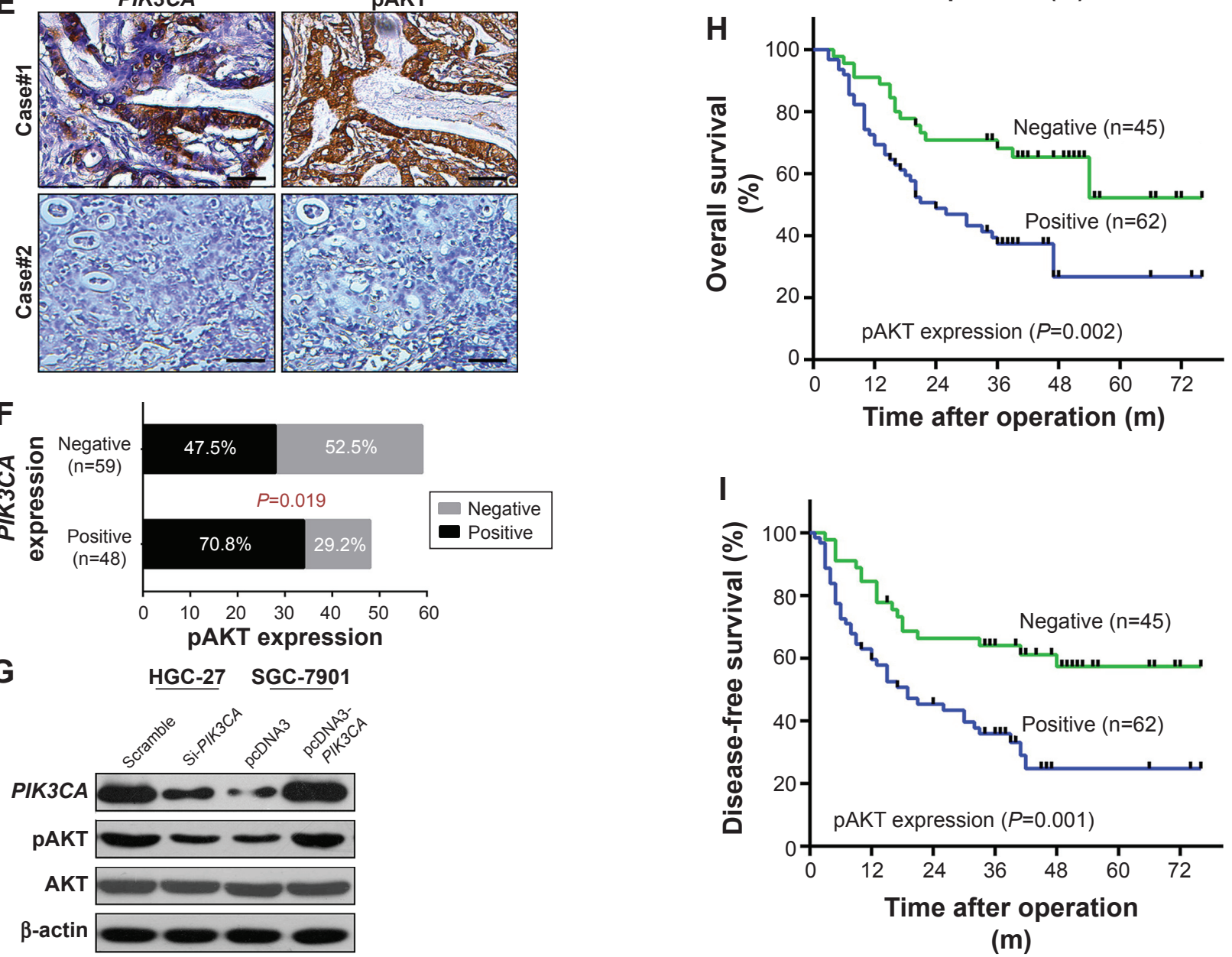

$(\mathrm{m})$

Figure 3 PIK3CA expression is associated with miR203 and phosphorylation of AKT.

Notes: (A) Expression of miR203 in 107 pairs of gastric carcinoma and adjacent nontumorous tissues. Expression levels of miR203 were normalized to the corresponding levels of U6. (B) PIK3CA expression level was negatively correlated with miR203 expression level. (C, D) Survival relevance analysis of miR203 expression in gastric carcinoma. (E) Serial sections of human gastric carcinoma tissue were subjected to immunohistochemistry (IHC) staining with antibodies against PIK3CA and PAKT. (F) The box plots show the correlation between PIK3CA and PAKT in 107 gastric cancer patients by IHC assays. (G) Western blot analyzed the relationship between PIK3CA and PAKT and showed that PIK3CA positively correlated with PAKT. (H, I) Comparison of overall survival (OS) and disease-free survival (DFS) by $\mathrm{PAKT}$. $P<0.05$. 
that miR203 targeted $P I K 3 C A$ and inhibited $P I K 3 C A$ expression. Next, analysis was conducted between two groups, one with high miR203 expressions, while the other with low miR203 expressions. It was found that patients with high miR203 expressions had much longer overall survival time and disease-free survival time than those with low miR203 expressions (Figure 3C and D).

It has been reported that $P I K 3 C A$ promotes phosphorylation of AKT in many types of cancer. ${ }^{14-16}$ Therefore, we detected the correlation between PIK3CA expression and AKT phosphorylation. IHC assays in serial sections of gastric cancer tissues indicated that overexpression of $P I K 3 C A$ positively correlated with AKT phosphorylation (Figure $3 \mathrm{E}$ and $\mathrm{F}$ ). Furthermore, in vitro experiments also indicated that downregulation of $P I K 3 C A$ was accompanied with decreased AKT phosphorylation and upregulation of $P I K 3 C A$ was accompanied with increased AKT phosphorylation (Figure 3G). Interestingly, AKT phosphorylation is also a predictor for gastric cancer patients, and high expression of pAKT correlated with poor prognosis (Figure $3 \mathrm{H}$ and $\mathrm{I}$ ).

\section{MiR203 can suppress PIK3CA/AKT pathway}

To investigate the function of miR203 on $P I K 3 C A / A K T$ pathway in breast cancer cells lines, miR203 mimics were transfected into HGC27 cells. As shown in Figure 4A, miR203 mimics decreased expression of PIK3CA and pAKT. Furthermore, we observed that overexpression of $P I K 3 C A$ can abrogate the effect of miR203 on the expression of PIK3CA and pAKT (Figure 4A). We then confirmed that miR203 decreased colony formation and invasion ability of cells and overexpression of PIK3CA abrogated the effect of miR203 (Figure 4C and E).

In addition, we observed that miR203 ASO increased the expression of PIK3CA and pAKT, and knockdown of PIK3CA abrogated miR203 ASO induced increase of pAKT (Figure 4B). Colony formation and invasion assays of the cells further confirmed that miR203 facilitated the colony formation and invasion abilities, and knockdown of $P I K 3 C A$ by si-PIK3CA transfection rescued this process (Figure 4D and $\mathrm{F}$ ). Therefore, these results demonstrated that miR203 can suppress the $P I K 3 C A / A K T$ pathway.

\section{Discussion}

Gastric cancer is the second largest cause of cancer deaths worldwide. ${ }^{17}$ However, the underlying mechanisms involved in the development of gastric cancer have not been fully elucidated. Understanding the molecular mechanisms involved in the progression of gastric cancer will help in developing new diagnostic and therapeutic strategies. In recent years, many researchers have focused on signaling pathway deregulation in cancers. Among them, dysregulation of the $\mathrm{PI} 3 \mathrm{~K} / \mathrm{AKT}$ pathway in a wide spectrum of human cancers has become a research hot spot. ${ }^{13}$ PIK3CA, as an important component of PI3K, plays important role in PI3K/AKT signaling pathway. ${ }^{18}$

In this study, we found that PIK3CA is aberrantly expressed in gastric tissue and the aberrant expression of PIK3CA predicted poor prognosis of gastric cancer patients. Through bioinformatics analysis and experiments, we identified miR203 as an upstream molecular of $P I K 3 C A$ signaling pathway, which could inhibit PIK3CA expression. MiR203 is one kind of microRNAs (miRNAs), which is composed of 19-24 nucleotides and noncoding RNA. ${ }^{19}$ In recent years, growing evidence has suggested that dysregulation of miRNAs contributes to tumor genesis. ${ }^{20}$ Studies have shown that more than $50 \%$ of miRNAs are located in cancer-associated genomic regions or in fragile sites. ${ }^{21}$ MiR203 was found to be significantly downregulated during malignant transformation in a wide variety of tumors. ${ }^{22}$ In this study, we found miR203 expression level was downregulated in gastric cancer tissue, and thus induced overexpression of $P I K 3 C A$. The low expression of miR203 also predicted poor prognosis of gastric cancer. Moreover, we found miR203 exerted anti-cancer effect via inhibiting proliferation and invasion of gastric cancer cells. Further study suggested that the anti-cancer effect of miR203 is, at least partly, due to the inhibition of PIK3CA expression. On account of the correlation between PI3K and phosphorylation of AKT, we also detected phosphorylation level of AKT in gastric cancer tissues and cells that knocked down or overexpressed $P I K 3 C A$. Interestingly, we found that $P I K 3 C A$ expression level positively correlated with phosphorylation of AKT. These results suggested that there is a miR203-PIK3CA-AKT signaling pathway in gastric cancer cells. MiR203 down-regulation induced overexpression of PIK3CA and thus promoting phosphorylation of AKT, the important effector downstream of PI3K. This pathway might play an important role in genesis and development of gastric cancer, which needs our further investigation in detail.

In conclusion, the current study illustrated that downregulation of miR203 and overexpression of $P I K 3 C A$ were novel potential prognosis predictors of gastric cancer patients. MiR203 as a cancer suppresser can inhibit proliferation and invasion of gastric cancer cells through downregulating $P I K 3 C A$. The results of our study will provide new diagnosis and therapeutic targets of gastric cancer. 
A

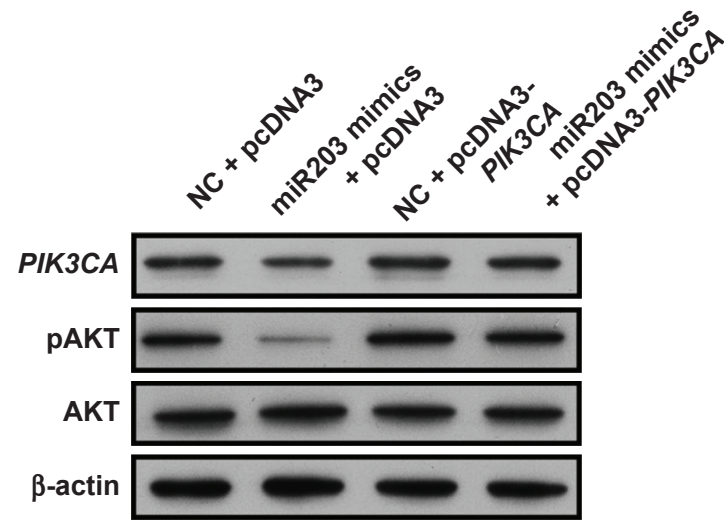

C
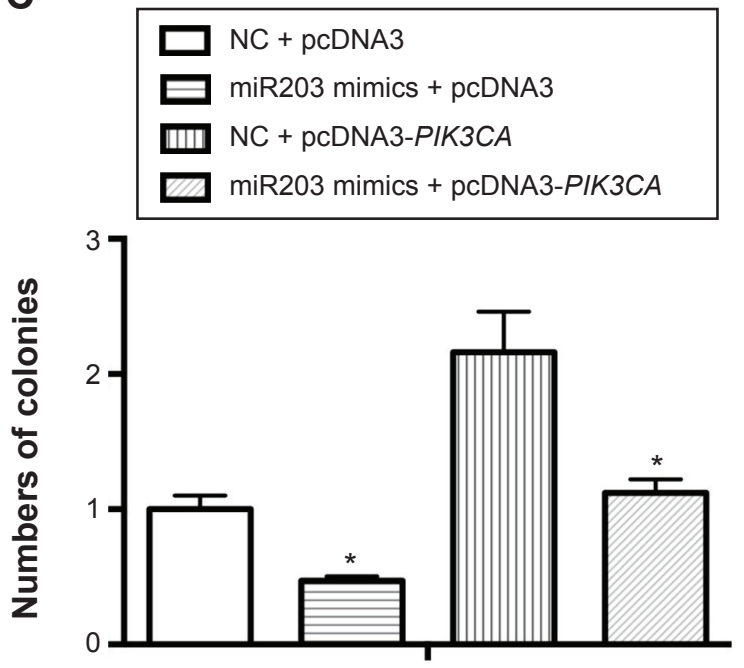

HGC-27

E
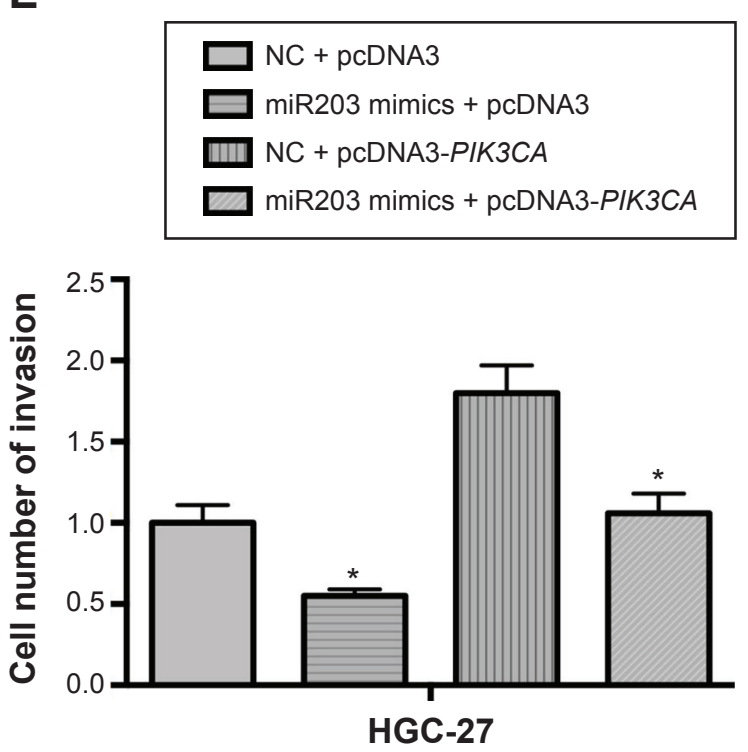

B

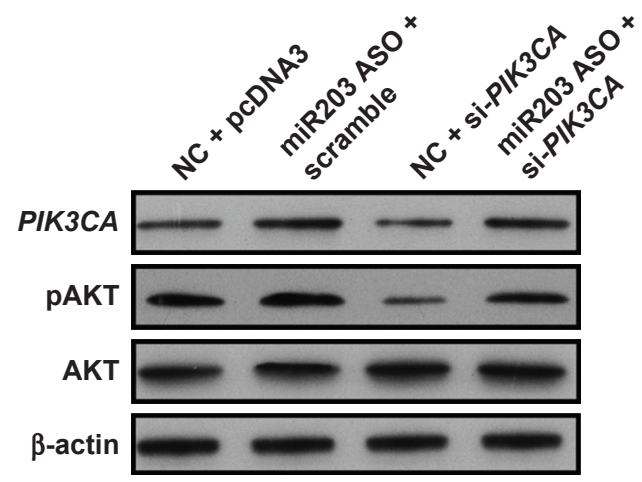

D
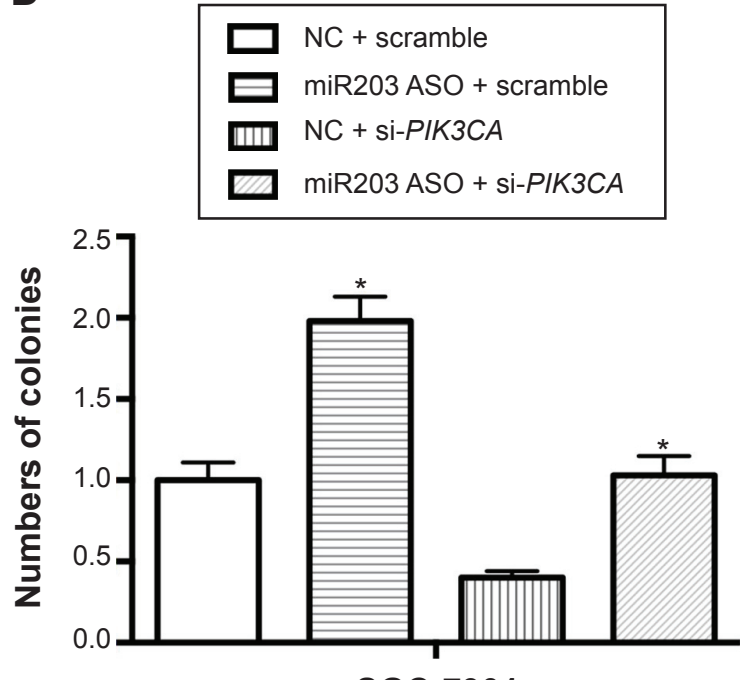

SGC-7901

$\mathbf{F}$
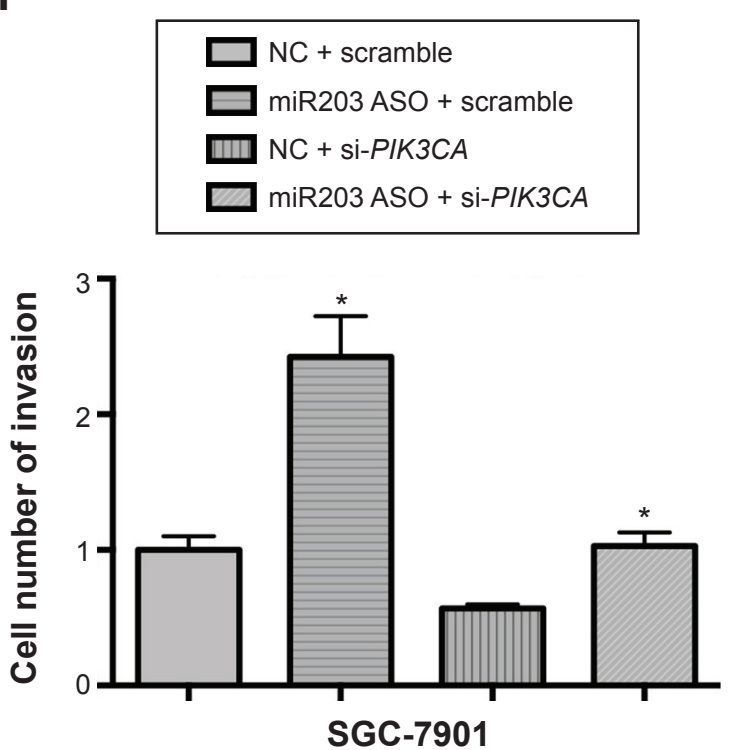

Figure 4 MiR203 can suppress PIK3CA/AKT pathway.

Notes: (A) Western blot was performed to detect the effect of miR203 mimics or pcDNA3-PIK3CA on the expression of PIK3CA and pAKT. (B) Western blot was performed to detect the effect of miR203 ASO or si-PIK3CA on the expression of PIK3CA and pAKT. (C, D) Colony formation or transwell invasion assays were performed to detect the effect of miR203 mimics or pcDNA3-PIK3CA on the colony formation or transwell invasion abilities. (E, F) Colony formation or transwell invasion assays were performed to detect the effect of miR203 ASO or si-PIK3CA on the colony formation or transwell invasion abilities. ${ }^{*} P<0.05$ represents the values compared to the NC $+p c D N A 3 . I$ or scramble. Abbreviation: NC, normal control. 


\section{Acknowledgments}

This work was supported by the grant from Educational Commission of GuangDong Province, People's Republic of China (No 2013KJCX0153), GuangDong Provincial Department of Science and Technology, People's Republic of China (No 2012B031800432) and Guangzhou Department of Science and Information Technology, People's Republic of China (No 2014Y2-00092).

\section{Disclosure}

The authors report no conflicts of interest in this work.

\section{References}

1. Zhang L, Tian W, Kim S, Ding W, Tong Y, Chen S. Arsenic sulfide, the main component of realgar, a traditional Chinese medicine, induces apoptosis of gastric cancer cells in vitro and in vivo. Drug Des Devel Ther. 2014;9:79-92.

2. Chen W, Zheng R, Zhang S, et al. Report of incidence and mortality in China cancer registries, 2009. Chin J Cancer Res. 2013;25:10-21.

3. Coburn NG. Lymph nodes and gastric cancer. J Surg Oncol. 2009;99: 199-206.

4. Song KY, Park SM, Kim SN, Park CH. The role of surgery in the treatment of recurrent gastric cancer. Am J Surg. 2008;196:19-22.

5. Volinia S, Hiles I, Ormondroyd E, et al. Molecular cloning, cDNA sequence, and chromosomal localization of the human phosphatidylinositol 3-kinase p110 alpha (PIK3CA) gene. Genomics. 1994;24: 472-477.

6. Samuels Y, Wang Z, Bardelli A, et al. High frequency of mutations of the PIK3CA gene in human cancers. Science. 2004;304:554.

7. Bachman KE, Argani P, Samuels Y, et al. The PIK3CA gene is mutated with high frequency in human breast cancers. Cancer Biol Ther. 2004;3: $772-775$.

8. Li VS, Wong CW, Chan TL, et al. Mutations of PIK3CA in gastric adenocarcinoma. BMC Cancer. 2005;5:29.

9. Lin Y, Jiang X, Shen Y, et al. Frequent mutations and amplifications of the PIK3CA gene in pituitary tumors. Endocr Relat Cancer. 2009; $16: 301-310$.
10. Mao C, Yang ZY, Hu XF, Chen Q, Tang JL. PIK3CA exon 20 mutations as a potential biomarker for resistance to anti-EGFR monoclonal antibodies in KRAS wild-type metastatic colorectal cancer:a systematic review and meta-analysis. Ann Oncol. 2012;23:1518-1525.

11. Liao X, Morikawa T, Lochhead P, et al. Prognostic role of PIK3CA mutation in colorectal cancer: cohort study and literature review. Clin Cancer Res. 2012;18:2257-2268.

12. Lievre A, Blons H, Laurent-Puig P. Oncogenic mutations as predictive factors in colorectal cancer. Oncogene. 2010;29:3033-3043.

13. Zhou XL, Tang SS, Yi G, et al. RNAi knockdown of PIK3CA preferentially inhibits invasion of mutant PIK3CA cells. World J Gastroenterol. 2011;17:3700-3708.

14. Liu JF, Zhou XK, Chen JH, et al. Up-regulation of PIK3CA promotes metastasis in gastric carcinoma. World J Gastroenterol. 2010;16: 4986-4991.

15. Nyakern M, Tazzari PL, Finelli C, et al. Frequent elevation of Akt kinase phosphorylation in blood marrow and peripheral blood mononuclear cells from high-risk myelodysplastic syndrome patients. Leukemia. 2006;20:230-238.

16. Jiang H, Gao M, Shen Z, et al. Blocking PI3K/Akt signaling attenuates metastasis of nasopharyngeal carcinoma cells through induction of mesenchymal-epithelial reverting transition. Oncol Rep. 2014;32:559-566.

17. Lu J, Huang CM, Zheng CH, et al. Analysis on the clinical and pathological features and prognosis of familial gastric cancer in South china population: a single-center study of 724 patients. J Oncol. 2012;2012: 641218.

18. Fresno Vara JA, Casado E, de Castro J, Cejas P, Belda-Iniesta C, GonzalezBaron M.PI3K/Akt signalling pathway and cancer. Cancer Treat Rev.2004; 30:193-204.

19. Miranda KC, Huynh T, Tay Y, et al. A pattern-based method for the identification of MicroRNA binding sites and their corresponding heteroduplexes. Cell. 2006;126:1203-1217.

20. Bartel DP. MicroRNAs: genomics, biogenesis, mechanism, and function. Cell. 2004;116:281-297.

21. Yang H, Fang F, Chang R, Yang L. MicroRNA-140-5p suppresses tumor growth and metastasis by targeting transforming growth factor beta receptor 1 and fibroblast growth factor 9 in hepatocellular carcinoma. Hepatology. 2013;58:205-217.

22. Yuan Y, Zeng ZY, Liu XJ, et al. MicroRNA-203 inhibits cell proliferation by repressing DeltaNp63 expression in human esophageal squamous cell carcinoma. BMC Cancer. 2011;11:57.
Drug Design, Development and Therapy

\section{Publish your work in this journal}

Drug Design, Development and Therapy is an international, peerreviewed open-access journal that spans the spectrum of drug design and development through to clinical applications. Clinical outcomes, patient safety, and programs for the development and effective, safe, and sustained use of medicines are a feature of the journal, which

\section{Dovepress}

has also been accepted for indexing on PubMed Central. The manuscript management system is completely online and includes a very quick and fair peer-review system, which is all easy to use. Visit http://www.dovepress.com/testimonials.php to read real quotes from published authors. 